\title{
Monte Carlo Simulations of Ultrathin Magnetic Dots
}

\author{
M. Rapini, R. A. Dias, B. V. Costa, \\ Laboratório de Simulação - Departamento de Física - ICEX - UFMG 30123-970 Belo Horizonte - MG, Brazil \\ and D. P. Landau \\ Center for Simulational Physics, University of Georgia, Athens, Georgia 30602
}

Received on 19 September, 2005

\begin{abstract}
In this work we study the thermodynamic properties of ultrathin ferromagnetic dots using Monte Carlo simulations. We investigate the vortex density as a function of the temperature and the vortex structure in monolayer dots with perpendicular anisotropy and long-range dipole interaction. The interplay between these two terms in the hamiltonian leads to an interesting behavior of the thermodynamic quantities as well as the vortex density.
\end{abstract}

Keywords: Monte Carlo; Ultrathin films; Phase transition

\section{INTRODUCTION}

Magnetism at nanoscale, when the size of the structure is comparable to or smaller than both the ferromagnetic (FM) and antiferromagnetic (AF) domain size, offers a great potential for new physics. In the last decade there has been an increasing interest in ultrathin magnetic dots from research groups as well as technological industries. Such an interest is due to numerous unique phenomena related to the lowdimension of these systems.

The modern technology demands techniques capable of producing nanometer-sized structures over large areas. A good perspective is the use of nanodots of nickel that could store terabyte of data in a computer chip just a few centimeters wide. In particular, ferromagnetic nanodots have been widely studied by use of experimental techniques such as MFM (magnetic force microscopy). In addition, some theoretical models were proposed to explain the physical phenomena observed in the experiments, among them the transition from perpendicular to in-plane ordering and the magnetoresistence effect.

Regarding the perpendicular to in-plane ordering transition, experiments were done using epitaxial films to investigate its transition temperature and thickness dependence [2] [3]. In addition, many theoretical approaches were developed, for example, treating a two-dimensional layer by renormalization group [4]. Some lattice models were proposed to take into account long-range dipolar interactions and surface anisotropy [5].

Based on such models, Monte Carlo simulations have been widely used to study the phase diagram of very thin films [6], the nature of this transition [7] as well as its dependence on the magnetic history of the system [8]. On the other hand, magnetic domains [9] and magnetic structures [10] have also been investigated by using computational methods. A topological excitation, the spin vortex, has been found in experiments and also detected in simulations. Vortex structures are believed to drive a Bereziinski-Kosterlitz-Thouless (BKT) phase transition in the two dimensional planar-rotator (PR) model [11]. Although vortices are present in thin films with long range interactions, it is not clear if they play any role in the transition.

The model we study is described by the Heisenberg spin hamiltonian with exchange and long-range dipolar interactions as well as single-ion anisotropy

$$
H=-J \sum_{<i j>} \mathbf{S}_{\mathbf{i}} \cdot \mathbf{S}_{\mathbf{j}}+\mathrm{D} \sum_{\mathrm{i} \neq \mathrm{k}} \frac{\mathbf{S}_{\mathbf{i}} \cdot \mathbf{S}_{\mathbf{k}}}{\mathrm{r}_{\mathrm{ik}}^{3}}-3 \frac{\left(\mathbf{S}_{\mathbf{i}} \cdot \mathbf{r}_{\mathbf{i k}}\right)\left(\mathbf{S}_{\mathbf{k}} \cdot \mathbf{r}_{\mathbf{i k}}\right)}{\mathrm{r}_{\mathrm{ik}}^{5}}-\mathrm{A} \sum_{\mathrm{i}}\left(\mathrm{S}_{\mathrm{i}}^{\mathrm{z}}\right)^{2}
$$

where we use classical spins $|\mathbf{S}|=1$. Here the first sum is performed over nearest neighbors with exchange coupling strenght, $J>0$, while the second sum runs over all spin pairs in the lattice. The constant of dipole coupling is $D, \mathbf{r}_{i k}$ is a vector connecting the $i$ and $k$ sites and $A$ is the single-site anisotropy constant along the $\mathrm{z}$-axis[5].

The main task in this work is to study the importance of vortices in the physics of the model. Although preliminary, our results indicate an anomalous behavior of the vortex density at the transition temperature for $\delta=\frac{D}{A} \ll 1$. In the following we present a brief background on the simulation, our results and the conclusions.

\section{Method}

The simulations are done in a square lattice of volume $L \times L$ with $L=20,40,60$ by using the Monte-Carlo method with the Metropolis algorithm [12, 13]. Since nanodots are finite per nature we have to use open boundary conditions in our simulations. However, we want to emphasize the long range effects of the dipolar term of the model at the boundary of the structure. For that, we have used periodic boundary 
conditions in the non dipolar terms while for the dipolar term we have used open conditions.

We have studied the model for three different values of the parameters $A$ and $D, \delta=\frac{D}{A}=0.1,1.0$ and 9.0 for fixed $J=1$. Energy is measured in units of $J S^{2}$ and temperature in units of $J S^{2} / k_{B}$, where $k_{B}$ is the Boltzman constant. For every temperature the first $10^{5} \mathrm{MC}$ steps per spin were used to lead the system to equilibrium and the next $10^{5}$ configurations were used to calculate thermal averages of thermodynamical quantities of interest.

\section{RESULTS}

In the case where $\delta=0.1$, we measured the out-of-plane $(z)$ and in-plane ( $x y)$ magnetizations (Shown in Fig. 1).

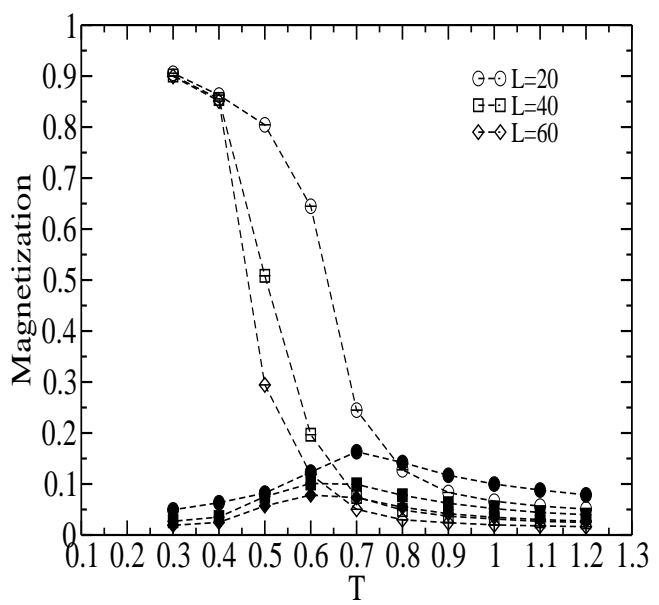

FIG. 1: Out-of-plane and in-plane magnetization (open and full symbols) for $\delta=0.1$

The system comes from an ordered state at low temperature to a disordered state at high temperature. That behavior indicates an order-disorder phase transition at $T_{c} \approx 0.55$. The in-plane magnetization, $M_{x y}$, grows presenting a maximum close to the order-disorder critical temperature $T_{c}$. However, the height of the peak diminishes as $L$ grows, in a clear indicative that it is a finite size artifice.

The magnetic susceptibility is shown in Fig. 2. The position of the maxima give us an estimate for $T_{c}(\approx 0.55)$.

We also measured the vortex density in the $x y$ plane as a function of the temperature. Starting from the highest temperature, $T=1.2$, the number of vortex decreases and reaches a minimum. Then it starts to increase as the system is cooled down. This behavior is shown in Fig. 3 and the graphics indicate that the ground state of the system has a significant number of vortices and anti-vortices in the $x y$ plane. Apparently, the minimum of the vortex curve is connected with the transition to in-plane magnetization, however, we were not able to establish that connection.

For $\delta=1.0$ the behavior of the in-plane and out-of-plane magnetizations (See Fig. 4), suggest that the ground state is

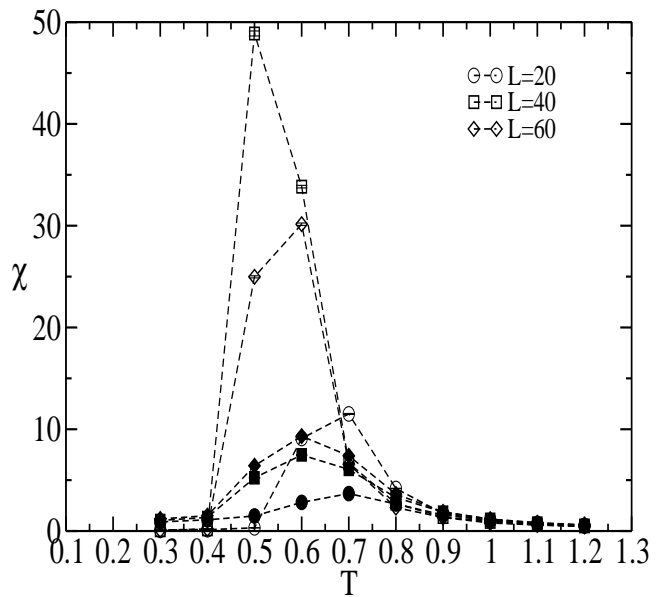

FIG. 2: Out-of-plane (open symbols) and in-plane (full symbols) susceptibilities for $\delta=0.1$.

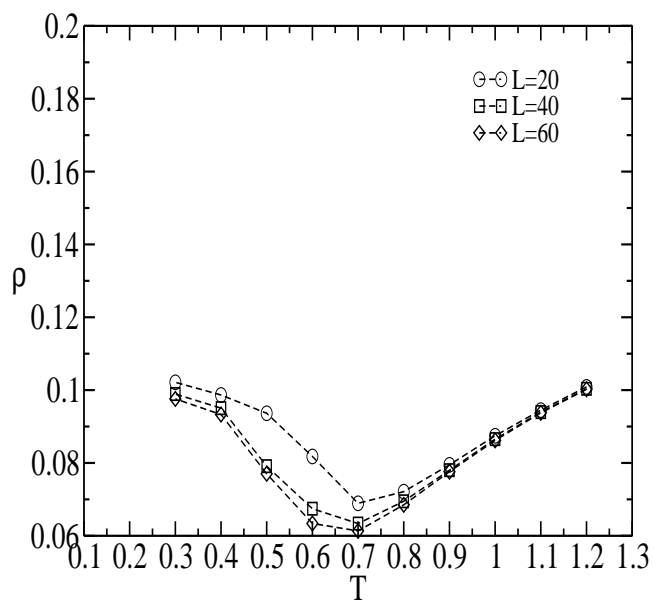

FIG. 3: Vortex density in the $x y$ plane for $\delta=0.1$.

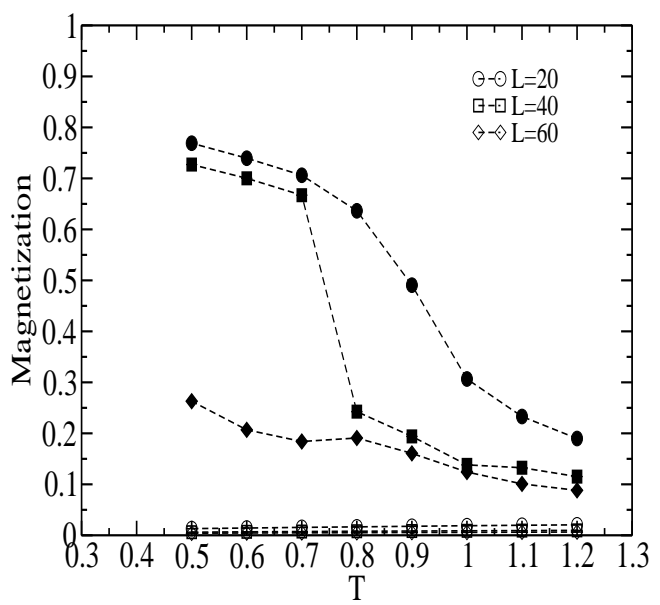

FIG. 4: Out-of-plane (open symbols) and in-plane (full symbols) magnetization for $\delta=1.0$ 
disordered in contrast to earlier works of Santamaria [6] and Vedmedenko [10] that argue that the ground state is for spins ordered in the $x y$ plane. A plot of the susceptibility is shown in Fig. 5 as a function of temperature. Although some authors $[6,10]$ concluded that this transition is of second order, the curves show well defined maxima that do not seem to indicate any critical behavior.

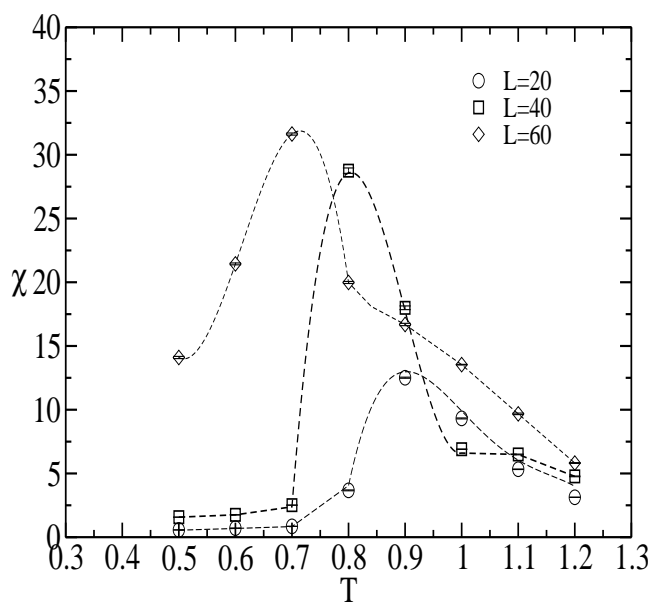

FIG. 5 : In-plane susceptibility for $\delta=1.0$. The lines are only a guide to the eye.

The vortex density curve in the $x y$ plane is shown in Fig. 6 . We see that the number of vortices increases monotonically from zero as a function of temperature. As temperature grows we observed that the spins in the lattice start to disorder, so that pairs vortices-anti-vortices can unbind inducing a $B K T$ transition. However our results are not refined enough to decide that. In Fig. 7 we show two typical configurations for $T=0.8$ and 1.2 where the vortices are indicated by circles and the anti-vortices by squares.

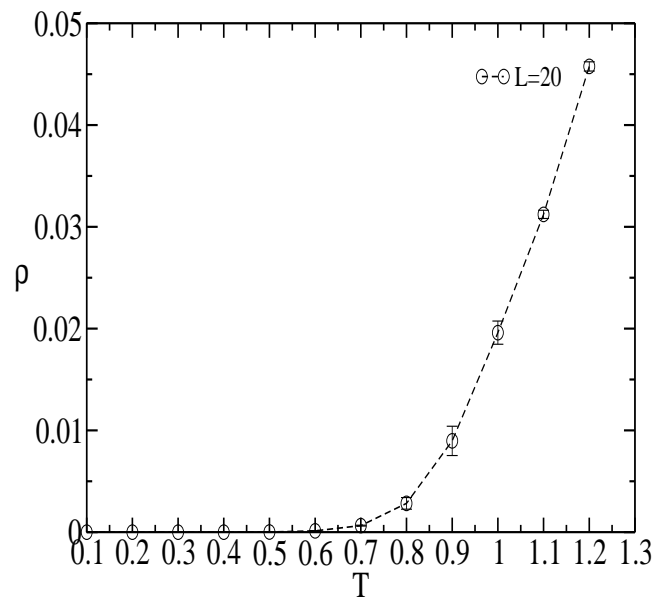

FIG. 6: Vortex density in the $x y$-plane for $\delta=1.0$.

For systems with larger $\delta$, for example, $\delta=9.0$, the spins are preferentially in the $x y$ plane but it does not present any

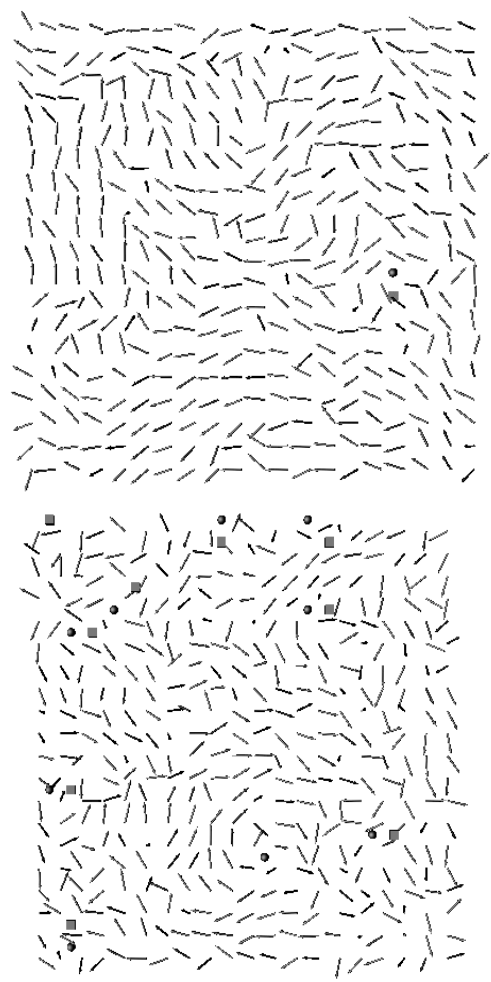

FIG. 7: Configurations of the system with $\delta=1.0$ for $T=0.8 \mathrm{e}$ $T=1.2$. The vortices are indicated by spheres and the anti-vortices by cubes.

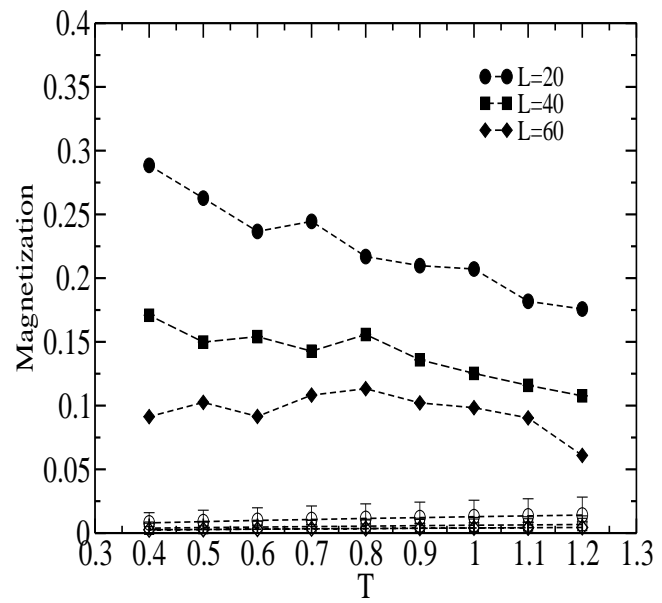

FIG. 8: Out-of-plane and in-plane magnetization (open and full symbols) for $\delta=9.0$

magnetic ordering (See Fig. 8). The vortex density curve is similar to the case where $\delta=1.0$ (See Fig. 9). 


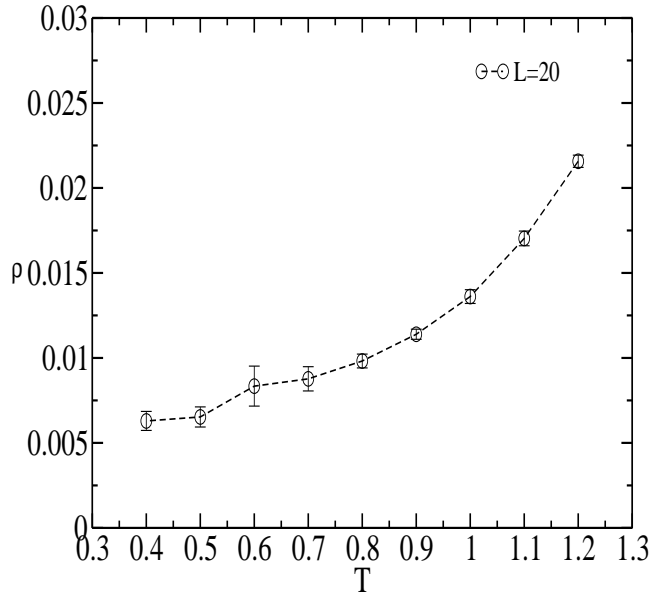

FIG. 9: Vortex density in the $x y$-plane for $\delta=9.0$.

\section{CONCLUSION}

In summary, we investigated the Heisenberg spin model with exchange $J$ and dipolar interactions $D$ and an anisotropic term $A$ for different parameters $\delta=\frac{D}{A}$. For small $\delta$, $(0.1)$, we observed that the vortex density has a minimum and is nonzero for low temperatures. Apparently, this minimum is connected with the order-disorder phase transition but this connection has to be studied more carefully. For larger values of $\delta$ (1.0 and 9.0) the vortex density and the configurations of vortices in the system led us to suspect of a phase transition of the BKT type involving the unbinding of vortices-antivortices pairs. However our results are not refined enough to decide that.

\section{Acknowledgments}

BVC would like to thank the Center for Simulational Physics at the University of Georgia for its hospitality. This work was funded by $\mathrm{CNPq}$ and FAPEMIG, Brazilian agencies, and CIAM-CNPq Process Grant No. 49.0101/03-8, NASA Grant No. NNC04GB24 and NSF Grants Nos. DMR0341874 and DMR-0307082.
[1] Electronic mail: mrapini@ fisica.ufmg.br

[2] R. Allenspach, A. Bischof, Phys. Rev. Lett. 69, 3385 (1992).

[3] D. P. Pappas et al. , J. Appl. Phys. 69, 5209 (1991).

[4] D. Pescia, V. L. Pokrovsky, Phys. Rev. Lett. 65, 2599 (1990).

[5] A. Moschel, K. D. Uasdel, Phys. Rev. B 49, 12868 (1994).

[6] C. Santamaria, H. T. Diep, J. Magn. Magn. Mat. 212, 23 (2000).

[7] A. Hucht, A. Moschel, and K. D. Usadel, J. Magn. Magn. Mat. 148, 32 (1995).

[8] O. Iglesias, A. Valencia, and A. Labarta, J. Magn. Magn. Mat. 196, 819 (1999).
[9] F. Matsubara, S. Endoh, J. Magn. Magn. Mat. 272, 679 (2004).

[10] E. Y. Vedmedenko, A. Ghazali, and J. C. S. Lévy, Surface Science 402-404, 391 (1998).

[11] J. B. Kogut, Rev. Mod. Phys 51, 659 (1979).

[12] J. M. Thijssen, Computational Physics, Cambridge University Press (1999).

[13] D. P. Landau and K. Binder, A Guide to Monte Carlo Simulations in Statistical Physics, Cambridge University Press, Cambridge (2000). 